\title{
$\beta$-Caryophyllene promotes osteoblastic mineralization, and suppresses osteoclastogenesis and adipogenesis in mouse bone marrow cultures in vitro
}

\author{
MASAYOSHI YAMAGUCHI ${ }^{1}$ and ROBERT M. LEVY ${ }^{2}$ \\ ${ }^{1}$ Department of Hematology and Medical Oncology, Emory University School of Medicine, Atlanta, GA 30322; \\ ${ }^{2}$ Department of Clinical Development, Primus Pharmaceuticals, Inc, Scottsdale, AZ 85253, USA
}

Received June 3, 2016; Accepted July 20, 2016

DOI: $10.3892 / \mathrm{etm} .2016 .3818$

\begin{abstract}
Osteoporosis is induced by the reduction in bone mass through decreased osteoblastic osteogenesis and increased osteoclastic bone resorption, and it is associated with obesity and diabetes. Osteoblasts and adipocytes are derived from bone marrow mesenchymal stem cells. The prevention of osteoporosis is an important public health concern in aging populations. $\beta$-caryophyllene, a component of various essential oils, is a selective agonist of the cannabinoid receptor type 2 and exerts cannabimimetic anti-inflammatory effects in animals. The present study aimed to identify the effect of $\beta$-caryophyllene on adipogenesis, osteoblastic mineralization and osteoclastogenesis in mouse bone marrow cell cultures in vitro. Bone marrow cells obtained from mouse femoral tissues were cultured in the presence of $\beta$-caryophyllene (0.1-100 $\mu \mathrm{M})$ in vitro. The results revealed that $\beta$-caryophyllene stimulated osteoblastic mineralization, and suppressed adipogenesis and osteoclastogenesis. Thus, $\beta$-caryophyllene may be used as a therapeutic agent for the prevention and treatment of osteoporosis.
\end{abstract}

\section{Introduction}

Bone homeostasis is maintained through osteocytes, osteoclasts and osteoblasts that are present in bone tissues $(1,2)$. Osteoblasts, which develop from bone marrow mesenchymal stem cells, promote bone formation and mineralization. By contrast, osteoclasts are derived from hematopoietic progenitor cells and stimulate bone resorption $(1,2)$. Bone marrow mesenchymal stem cells (MSCs) are multipotent stromal cells that can differentiate into a number of different cell types, including

Correspondence to: Dr. Masayoshi Yamaguchi, Department of Hematology and Medical Oncology, Emory University School of Medicine, Building C, 1365 Clifton Road, Atlanta, GA 30322, USA E-mail: yamamasa1155@yahoo.co.jp

Key words: $\beta$-caryophyllene, adipogenesis, osteoblastogenesis, osteoclastogenesis, bone marrow cells adipocytes, myoblasts, osteoblasts and chondrocytes $(3,4)$. MSC differentiation is triggered by crosstalk between complex signaling pathways involving numerous components, such as Wnt, delta/jagged, bone morphogenic, Wnt and hedgehog proteins, as well as insulin, insulin-like growth factors, fibroblastic growth factors, and transcriptional regulators of adipocyte and osteoblast differentiation, including peroxisome proliferators-activated receptor- $\gamma$ (PPAR $\gamma)$ and runt-related transcription factor 2 (5-7). Bone marrow MSC differentiation is crucial for the homeostasis of bone remodeling.

Osteoporosis is associated with a deterioration of bone mass through the suppression of osteoblastic osteogenesis and promotion of osteoclastic bone resorption, and may result in bone fractures (8). Osteoporosis is widely recognized as a major public health problem worldwide and the incidence is increasing in countries with ageing populations (8). Fractures of the proximal femur represent the most serious complication of this disease (8). Bone mass decrease in females is primarily due to reduced secretion of estrogen following the beginning of menopause (8); thus, osteoporosis is an important cause of morbidity and mortality. Furthermore, there is growing evidence that osteoporosis is associated with obesity and diabetes, which are increasingly becoming a major public health concern $(9,10)$. Notably, osteoporosis and obesity are implicated with a number of features $(3,4,11,12)$; osteoblasts and adipocytes develop from bone marrow MSCs and there is a reverse association between the differentiation of MSCs into osteoblasts and adipocytes. Since MSCs differentiate osteoblasts, the differentiation of osteoblasts into adipocytes is reduced $(3,4)$. In addition, a previous study identified that obesity, diabetes and osteoporosis were associated with bone marrow adiposity, which increases production of the inflammatory cytokine tumor necrosis factor- $\alpha$ (TNF- $\alpha$ ) (13). TNF- $\alpha$ has been shown to suppress osteoblastogenesis and bone mineralization $(14,15)$. These previous findings suggest that agents inhibiting adipogenesis and stimulating osteoblastogenesis will be useful in the prevention and treatment of osteoporosis.

Numerous constituents of herbs are known to possess anti-inflammatory and analgesic effects (16-19). The sesquiterpene $\beta$-caryophyllene is present in various essential oils, particularly in clove oil derived from the stems and flowers 
of Syzygium aromaticum, rosemary oil from Rosmarinus officinalis, hemp oil from Cannabis sativa, and in cinnamon and hop oils (16-19). $\beta$-caryophyllene is found in numerous edible plants that are ingested daily, and it is approved as a food additive by the Food and Drug Administration (FDA). It is a selective agonist of cannabinoid receptor type 2 (CB2) and exerts anti-inflammatory effects in animals $(17,19)$. In addition, $\beta$-caryophyllene reduces acute and chronic pain associated with inflammation (19-21). The anti-inflammatory effects of $\beta$-caryophyllene have been implicated with reduced TNF- $\alpha$ and interleukin (IL)- $1 \beta$ production, which is associated with opioid receptors (22).

Plant-derived molecules that inhibit adipogenesis and stimulate osteoblastic bone mineralization are poorly understood. The present study aimed to determine whether $\beta$-caryophyllene regulates the differentiation of bone marrow cells that are associated with adipogenesis and osteoblastogenesis. $\beta$-caryophyllene was demonstrated to enhance osteoblastogenesis, and suppress adipogenesis and osteoclastogenesis in mouse bone marrow cells in vitro. To the best of our knowledge, this is the first time that results concerning the role of $\beta$-caryophyllene in the differentiation of bone marrow MSCs have been reported.

\section{Materials and methods}

Reagents. Dulbecco's modified Eagle's medium (DMEM) and antibiotics (penicillin and streptomycin) were purchased from Thermo Fisher Scientific, Inc. (Waltham, MA, USA). Fetal bovine serum (FBS) was obtained from HyClone (GE Healthcare Life Sciences, Logan, UT, USA). $\beta$-caryophyllene was purchased from Cayman Chemical Company (Ann Arbor, MI, USA). TNF- $\alpha$, tartrate-resistant acid phosphatase (TRAP), insulin, dexamethasone, 3-isobutyl-1-methylxanthine (IBMX), Alizarin Red S, Oil Red O and other reagents were purchased from Sigma-Aldrich (Merck Millipore, Darmstadt, Germany). Insulin was dissolved in diluted acetic acid solution and other reagents were dissolved in $100 \%$ ethanol.

Experimental animals and bone marrow cell isolation. Female C57BL6 mice ( $\mathrm{n}=8$; age, 2 months; weight, 18-20 g), purchased from Charles River Laboratories (Wilmington, MA, USA), were housed in a pathogen-free facility, with a $12 \mathrm{~h}$ light/dark cycle, temperature/atomosphere and ad libitum access to feed and water. All protocols used in the current study were approved by the Institutional Animal Care and Use Committee at Emory University School of Medicine (Atlanta, GA, USA). Tissue from the femur and tibia was removed immediately following sacrifice with exposure to $\mathrm{CO}_{2}$ in chamber box, and bone marrow cells were isolated from these tissues with needle flush under sterile conditions in safety cabinet $(23,24)$.

In vitro adipogenesis assay. This experiment was based on the methods described in our previous studies $(23,24)$. Bone marrow cells $\left(1 \times 10^{6}\right.$ cells/well; $2 \mathrm{ml}$ medium added per well using 12-well plates) were cultured in a water-saturated atmosphere containing $5 \% \mathrm{CO}_{2}$ and $95 \%$ air at $37^{\circ} \mathrm{C}$ for 3 days in culture medium, consisting of DMEM supplemented with $10 \%$ FBS and $1 \%$ penicillin-streptomycin (10,000 units/1). The cells were cultured in the presence or absence of differentiation medium (DM) $(23,24)$, which consisted of dexamethasone $(1 \mu \mathrm{M} / \mathrm{ml})$ and IBMX $(0.5 \mathrm{mM} / \mathrm{ml})$. Cells were treated with culture medium only, DM plus ethanol (final concentration, $0.1 \%$ ) or DM plus $\beta$-caryophyllene (0.1-100 $\mu \mathrm{M})$. Subsequently, the medium was replaced with fresh culture medium containing insulin $(10 \mu \mathrm{g} / \mathrm{ml})$ without dexamethasone and IBMX, and cells were cultured for a further 4 days in the presence or absence of $\beta$-caryophyllene (0.1-100 $\mu \mathrm{M})$. In other experimental groups, the cells were cultured in culture medium only, DM plus vehicle $(0.1 \%$ ethanol as a final concentration) or DM plus $\beta$-caryophyllene $(0.1-100 \mu \mathrm{M})$ for 3 days, then the medium was replaced with fresh culture medium containing insulin $(10 \mu \mathrm{g} / \mathrm{ml})$ and cultured for a further 4 days. The medium was then removed, and adipocytes were stained with Oil Red O. Cell numbers were counted under a light microscope (Olympus MTV-3; Olympus, Tokyo, Japan) using a Hemocytometer plate (23). Quantification was performed by extracting the dye with $0.2 \mathrm{ml}$ of isopropanol for $1 \mathrm{~min}$ and measuring the absorbance at $490 \mathrm{~nm}$ with a Spectracount microplate photometer. Results are presented as the mean \pm standard deviation of 8 replicate samples per data set using different dishes and cell preparations.

In vitro mineralization assay. Bone marrow cells $\left(1 \times 10^{6}\right.$ cells $/ \mathrm{ml} /$ well $)$ were cultured in 12 -well plates in the presence or absence of DMEM-mineralization medium [DMEM-MM; culture medium plus ascorbic acid $(100 \mu \mathrm{g} / \mathrm{ml})$ and $\beta$-glycerophosphate $(4 \mathrm{mM})]$ along with the vehicle or $\beta$-caryophyllene $(0.1-100 \mu \mathrm{M})(23,24)$, for 7 or 18 days at $37^{\circ} \mathrm{C}$ and $5 \% \mathrm{CO}_{2}$. The medium was changed every 3 days. After 18 days of culture, the cells were washed with phosphate-buffered saline (PBS) and stained with Alizarin Red S. For quantification, the dye was eluted with $10 \%$ cetylpyridinium chloride solution and the absorbance of the eluted solution at $570 \mathrm{~nm}$ was measured using a plate reader. Results were presented as the mean \pm standard deviation of 8 replicate samples per data set using different dishes and cell preparation.

In vitro osteoclastogenesis assay. Bone marrow cells $\left(2 \times 10^{5}\right.$ cells $/ \mathrm{ml} /$ well) were plated in 24-well plates with culture media ( $1 \mathrm{ml} /$ well) in an atmosphere containing $5 \% \mathrm{CO}_{2}$ at $37^{\circ} \mathrm{C}$. Cells were cultured in medium only (containing $0.1 \%$ ethanol as a final concentration), $\beta$-caryophyllene (0.1-100 $\mu \mathrm{M})$ only, TNF- $\alpha$ ( $5 \mathrm{ng} / \mathrm{ml}$ medium) or TNF- $\alpha$ ( $5 \mathrm{ng} / \mathrm{ml}$ medium) plus $\beta$-caryophyllene (0.1-100 $\mu \mathrm{M})$ for 3 days. Next, $0.5 \mathrm{ml}$ of the old medium was replaced with fresh culture medium with or without TNF- $\alpha(5 \mathrm{ng} / \mathrm{ml})$, and in the presence or absence of $\beta$-caryophyllene $(0.1-100 \mu \mathrm{M})$. The cultures were then maintained for a further 4 days $(25,26)$. After a total of 7 days of culture, adherent cells were stained for tartrate-resistant acid phosphatase (TRAP; Sigma-Aldrich; Merck Millipore), a marker of osteoclasts (26). Briefly, cells were washed with PBS, fixed with $10 \%$ neutralized formalin-phosphate $(\mathrm{pH} 7.2)$ for $10 \mathrm{~min}$, dried and then stained with acetate buffer $(\mathrm{pH}$ 5.0) containing Naphthol AS-MX phosphate (Sigma-Aldrich; Merck Millipore) in the presence of sodium tartrate $(10 \mathrm{mM})$ for $90 \mathrm{~min}$ at room temperature. TRAP-positive multinucleated cells (MNCs with $\geq 3$ nuclei) were considered to be osteoclast-like cells, and the cells were counted using light 

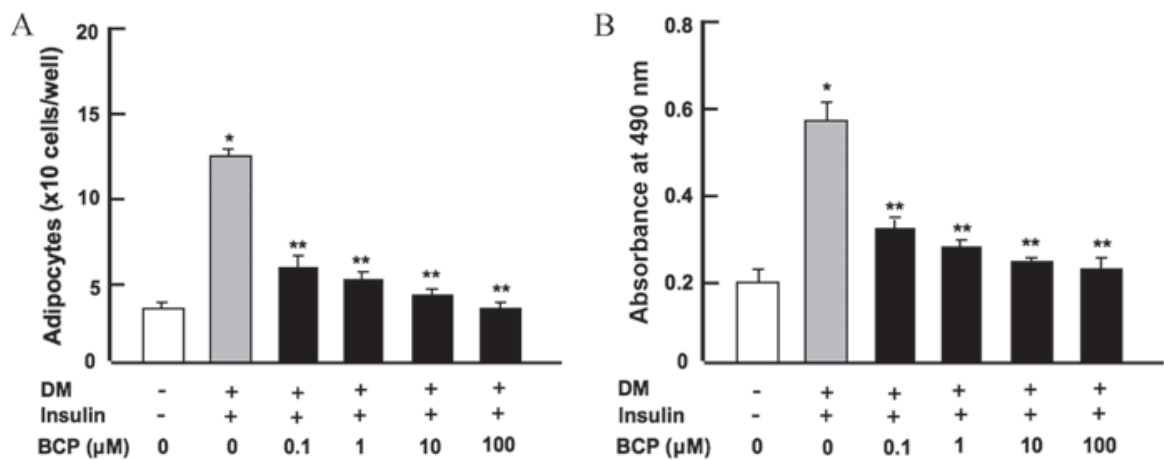

Figure 1. $\beta$-caryophyllene suppresses adipogenesis in mouse bone marrow cell cultures in vitro. (A) Number of adipocytes per cell, determined by counting under a light microscope. (B) Quantification of adipocytes based on the absorbance at $490 \mathrm{~nm}$ measured by spectrophotometry. Symbols indicate the presence $(+)$ and $(-)$ absence of the listed reagents. Statistical analysis was performed by one-way way analysis of the variance and post-hoc Tukey's range test. ${ }^{*} \mathrm{P}<0.001$ vs. control; ${ }^{* *} \mathrm{P}<0.001$ vs. DM + insulin only. DM, differentiation medium; $\mathrm{BCP}, \beta$-caryophyllene.

A

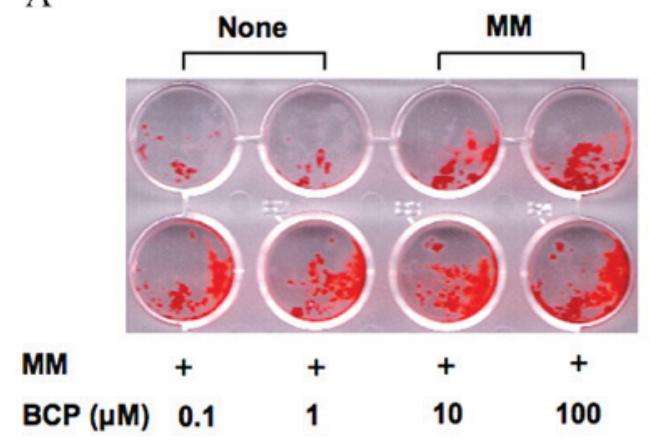

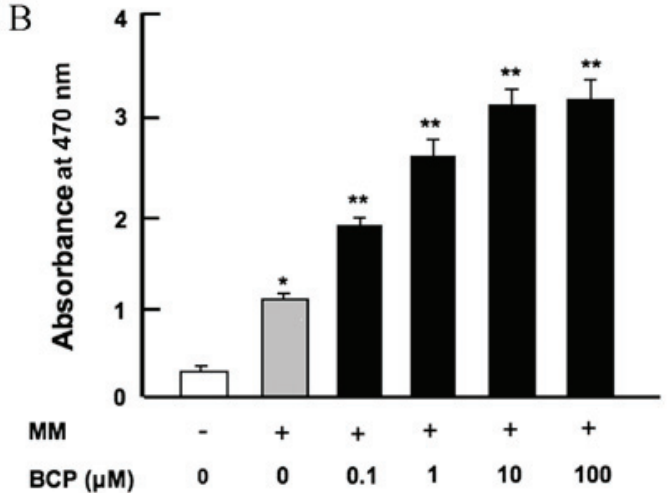

Figure 2. $\beta$-caryophyllene stimulates osteoblastic mineralization in mouse bone marrow cell cultures in vitro. (A) Representative image of cells cultured in none (without MM and $\beta$-caryophyllene) or MM in the presence or absence of increasing concentrations of $\beta$-caryophyllene $(0.1-100 \mu \mathrm{M})$ for 18 days. (B) Quantification of mineralization based on the absorbance at $570 \mathrm{~nm}$, determined by spectrophotometry. Symbols indicate the presence (+) and (-) absence of the listed reagents. The white bar represents the control cells grown in regular culture medium without MM or BCP. Statistical analysis was performed by one-way way analysis of the variance and post-hoc Tukey's range test. ${ }^{*} \mathrm{P}<0.001$ vs. control; ${ }^{* *} \mathrm{P}<0.001$ vs. MM only. MM, mineralization medium; $\mathrm{BCP}$, $\beta$-caryophyllene.

microscopy. MNC scores are expressed as the mean \pm standard deviation of six cultures with 2 replicate wells per data set using different dishes and cell preparation.

Statistical analysis. Statistical analysis was performed using GraphPad InStat software (version 3; GraphPad Software, Inc., La Jolla, CA, USA). Multiple comparisons were performed by one-way analysis of variance, followed by a post-hoc Tukey's range test for parametric data as indicated. $\mathrm{P}<0.05$ was considered to indicate a a statistically significant difference.

\section{Results}

$\beta$-caryophyllene suppresses bone marrow adipogenesis in vitro. Bone marrow MSCs are known to differentiate into adipocytes $(3,4)$. In order to determine the effect of $\beta$-caryophyllene on bone marrow adipogenesis, mouse bone marrow cells were cultured in either medium without DM and insulin or with DM plus insulin, along with $\beta$-caryophyllene $(0,0.1,1,10$ or $100 \mu \mathrm{M})$ for 7 days. Mouse bone marrow cells cultured without DM, insulin or $\beta$-caryophyllene acted as a control. As shown in Fig. 1, cells treated with DM + insulin alone significantly increased adipogenesis when compared with the control group (without DM or insulin; $\mathrm{P}<0.001$ ). The addition of $\beta$-caryophyllene at any concentration was observed to significantly suppress the differentiation of bone marrow cells into adipocytes ( $\mathrm{P}<0.001 \mathrm{vs}$. $\mathrm{DM}+$ insulin only) in a dose-dependent manner, as determined by cell counting (Fig. 1A) and spectrophotometry (Fig. 1B) following Oil $\mathrm{O}$ Red staining. Furthermore, reduced differentiation into adipocytes was found in bone marrow cells cultured in media containing $\beta$-caryophyllene for 3 days and then in media without $\beta$-caryophyllene for a further 4 days (data not shown).

\section{$\beta$-caryophyllene stimulates osteoblastic mineralization} in vitro. Osteoblasts develop from bone marrow MSCs $(3,4)$. To investigate the effect of $\beta$-caryophyllene on osteoblastogenesis and mineralization in the bone marrow, cells were cultured in $\mathrm{MM}$ with or without $\beta$-caryophyllene (0.1-100 $\mu \mathrm{M})$ for 18 days. As shown in Fig. 2, MM alone significantly increased bone marrow mineralization compared with the control group without MM $(\mathrm{P}<0.001)$. The addition of $\beta$-caryophyllene at all doses significantly increased osteoblastic mineralization in a dose-dependent manner when compared with cells treated with $\mathrm{MM}$ alone $(\mathrm{P}<0.001$; Fig. 2). Furthermore, increased osteoblastic mineralization was observed when bone marrow 

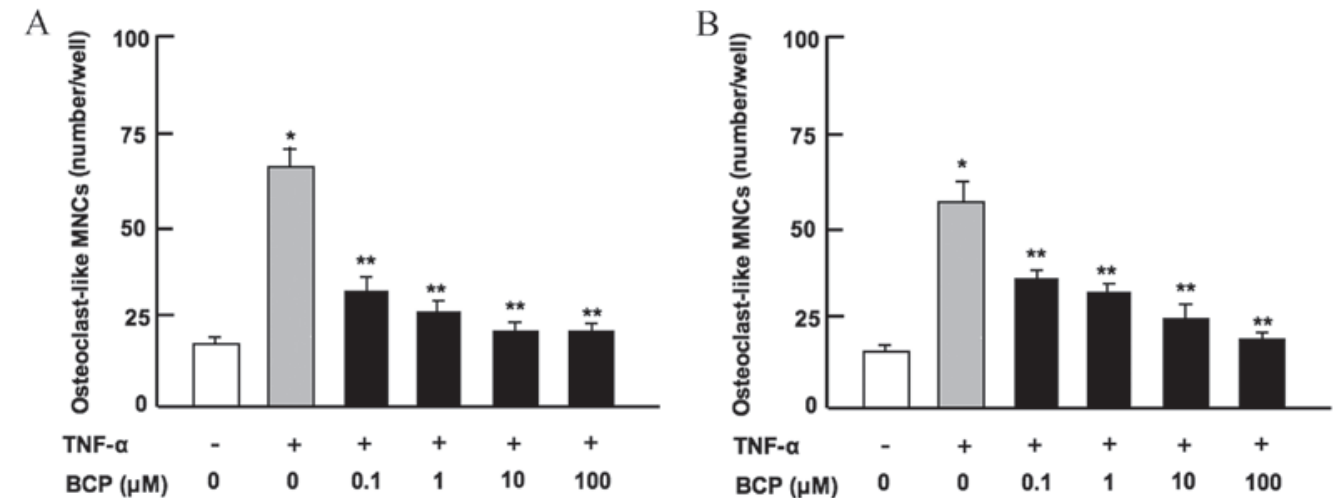

Figure 3. $\beta$-caryophyllene suppresses osteoclastogenesis in mouse bone marrow cell cultures in vitro. (A) Number of osteoclast-like MNCs, when bone marrow cells were cultured with TNF- $\alpha$ and increasing concentrations of $\beta$-caryophyllene $(0-100 \mu \mathrm{M})$ for 7 days. (B) Number of osteoclast-like MNCs, when bone marrow cells were cultured with TNF- $\alpha$ and $\beta$-caryophyllene $(0-100 \mu \mathrm{m})$ for 3 days, and then with TNF- $\alpha$ but without $\beta$-caryophyllene for a further 4 days. The white bar indicates the control and represents cells grown in regular culture medium without TNF- $\alpha$ or BCP. Statistical analysis was performed by one-way way analysis of the variance and post-hoc Tukey's range test. " $\mathrm{P}<0.001$ vs. control; ${ }^{* *} \mathrm{P}<0.001 \mathrm{vs}$. TNF- $\alpha$ alone. TNF- $\alpha$; tumor necrosis factor- $\alpha$; BCP, $\beta$-caryophyllene; MNCs, multinucleated cells.

cells were cultured in the presence of $\beta$-caryophyllene for 7 days (data not shown).

$\beta$-caryophyllene reduces osteoclastogenesis in vitro. Osteoclasts are differentiated from monocytes and macrophages in the bone marrow (26). To assess the effect of $\beta$-caryophyllene on osteoclastogenesis in bone marrow in vitro, bone marrow cells were cultured in the presence of TNF- $\alpha$, which stimulates osteoclastogenesis by activating

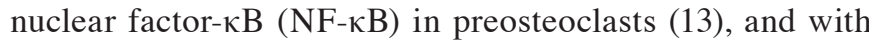
or without $\beta$-caryophyllene for 7 days. The results revealed that osteoclastogenesis was significantly enhanced in the presence of TNF- $\alpha$ alone when compared with the untreated control group $(\mathrm{P}<0.001 ;$ Fig. 3$)$. This increase was significantly suppressed following culture with $\beta$-caryophyllene at all doses for 7 days $(\mathrm{P}<0.001$ vs. TNF- $\alpha$ only; Fig. 3A). Furthermore, $\beta$-caryophyllene (0.1-100 $\mu \mathrm{M})$ did not have a significant effect on osteoclastogenesis in the absence of TNF- $\alpha$ (data not shown).

\section{Discussion}

In the present study, $\beta$-caryophyllene was demonstrated to enhance osteoblastic mineralization, and to suppress adipogenesis and osteoclastogenesis in mouse bone marrow cell cultures in vitro. To the best of our knowledge, this is the first time that these effects of $\beta$-caryophyllene are reported. The effects of $\beta$-caryophyllene on adipogenesis and osteoblastic mineralization were observed at an early stage of culture following 7-8 days. This indicates that $\beta$-caryophyllene strongly stimulates the differentiation of bone marrow MSCs into osteoblasts, and strongly suppresses the differentiation to adipocytes.

Bone marrow MSCs are multipotent cells that can differentiate into adipocytes and osteoblasts $(3,4)$. This process is mediated through numerous complex signaling pathways, including those involving PPAR $\gamma$ (5-7). For instance, enhanced mitogen-activated protein kinase/extracellular signal-regulated kinase signaling during adipogenesis potentiates the activity of factors that regulate the expression of CCAAT/enhancer-binding protein a and $\operatorname{PPAR} \gamma(6,7)$.
Similarly, $\beta$-caryophyllene may exhibit a specific regulatory effect on signaling pathways involved in the differentiation of bone marrow MSCs to adipocytes.

Osteoclasts, which promote bone resorption, are derived from hematopoietic progenitors $(1,2,26)$. The current study demonstrated that $\beta$-caryophyllene suppressed TNF- $\alpha$-enhanced osteoclastogenesis in mouse bone marrow in vitro, mediated through the activation of NF- $\mathrm{\kappa B}$ signaling in preosteoclasts. This suppressive effect was observed following 7 days culture with bone marrow cells. Therefore, the results of the present study suggest that $\beta$-caryophyllene inhibited osteoclastogenesis at the stage of differentiation into preosteoclasts in bone marrow culture.

The sesquiterpene $\beta$-caryophyllene is present in various essential oils, particularly in clove, hemp, rosemary and hop oil (16-22). In addition, $\beta$-caryophyllene is found in plants that are ingested daily and has been approved as a food additive by the FDA (16-18). $\beta$-caryophyllene is a selective agonist of CB2 and has been shown to have anti-inflammatory effects in animals, which have been implicated with reduced TNF- $\alpha$ and IL-1 $\beta$ production associated with opioid receptors $(17,19,22)$. Furthermore, this molecule reduces acute and chronic pain associated with inflammation (19-21). In the current study, $\beta$-caryophyllene was demonstrated to enhance osteoblastic mineralization, and suppress adipogenesis and osteoclastogenesis in mouse bone marrow cells in vitro. These results indicate that $\beta$-caryophyllene stimulates osteoblastic bone formation and suppresses osteoclastic bone resorption, which may provide a means to prevent and treat osteoporosis.

Osteoporosis has been associated with obesity and diabetes $(9,10)$, which are increasingly prevalent public health concerns. Osteoporosis and obesity share a number of similar features $(3,4,11,12)$; bone marrow MSCs can differentiate into adipocytes and osteoblasts and enhanced adipogenesis may suppress osteoblastogenesis in bone marrow cells $(3,4)$. In the present study, $\beta$-caryophyllene was found to stimulate osteoblastogenesis and suppress adipogenesis in mouse bone marrow cell cultures in vitro. Therefore, $\beta$-caryophyllene may serve an important role in the prevention and treatment of osteoporosis associated with obesity and diabetes. 
In conclusion, the present study demonstrated that $\beta$-caryophyllene promotes osteoblastic mineralization, and reduces adipogenesis and osteoclastogenesis in mouse bone marrow cell cultures in vitro. These results indicate that $\beta$-caryophyllene may be a useful tool in the treatment of osteoporosis. Further studies into the effects of $\beta$-caryophyllene on bone remodeling should be performed to validate these effects in an in vivo environment and in models of osteoporosis.

\section{References}

1. Raggatt LJ and Partridge NC: Cellular and molecular mechanisms of bone modeling. J Biol Chem 285: 25103-25108, 2010.

2. Chambers TJ and Fuller K: How are osteoclasts induced to resorb bone? Ann N Y Acad Sci 1240: 1-6, 2011.

3. Minguell JJ, Erices A and Conget P: Mesenchymal stem cells. Exp Biol Med (Maywood) 226: 507-520, 2001.

4. Muruganandan S, Roman AA and Sinal CJ: Adipocyte differentiation of bone marrow-derived mesenchymal stem cells: Cross talk with the osteoblastogenesis program. Cell Mol Life Sci 66: 236-253, 2009

5. Laudes M: Role of WNT signaling in the determination of human mesenchymal stem cells into preadipocytes. J Mol Endocrinol 46: R65-R72, 2011.

6. Gharibi B, Abraham AA, Ham J and Evans BA: Adenosine receptor subtype expression and activation influence the differentiation of mesenchymal stem cells to osteoblasts and adipocytes J Bone Miner Res 26: 2112-2124, 2011.

7. Kawai $\mathrm{M}$ and Rosen CJ: PPAR $\gamma$ : A circadian transcription factor in adipogenesis and osteogenesis. Nat Rev Endocrinol 6: 629-636, 2010

8. Weitzmann MN and Pacifici R: Estrogen deficiency and bone loss: An inflammatory tale. J Clin Invest 116: 1186-1194, 2006

9. Leslie WD, Rubin MR, Schwartz AV and Kanis JA: Type 2 diabetes and bone. J Bone Miner Res 27: 2231-2237, 2012.

10. Nielson CM, Srikanth P and Orwoll ES: Obesity and fracture in men and women: An epidemiologic perspective. J Bone Miner Res 27: 1-10, 2012.

11. Gharibi B, Abraham AA, Ham J and Evans BA: Adenosine receptor subtype expression and activation influence the differentiation of mesenchymal stem cells to osteoblasts and adipocytes. J Bone Miner Res 26: 2112-2124, 2011.

12. Pirih F, Lu J, Ye F, Bezouglaia O, Atti E, Ascenzi MG, Tetradis S, Demer L, Aghaloo T and Tintut Y: Adverse effects of hyperlipidemia on bone regeneration and strength. J Bone Miner Res 27 : 309-318, 2012.

13. Li Y, Li A, Strait K, Zhang H, Nanes MS and Weitzmann MN: Endogenous TNFalpha lowers maximum peak bone mass and inhibits osteoblastic Smad activation through NF-kappaB. J Bone Miner Res 22: 646-655, 2007.
14. Chang J, Wang Z, Tang E, Fan Z, McCauley L, Franceschi R, Guan K, Krebsbach PH and Wang CY: Inhibition of osteoblastic bone formation by nuclear factor-kappaB. Nat Med 15: 682-689, 2009.

15. Cortez M, Carmo LS, Rogero MM, Borelli P and Fock RA: A high-fat diet increases IL-1, IL-6, and TNF- $\alpha$ production by increasing NF- $\kappa \mathrm{B}$ and attenuating PPAR- $\gamma$ expression in bone marrow mesenchymal stem cells. Inflammation 36: 379-386, 2013.

16. Ghelardini C, Galeotti N, Di Cesare Mannelli L, Mazzanti G and Bartolini A: Local anaesthetic activity of beta-caryophyllene. Farmaco 56: 387-389, 2001.

17. Gertsch J, Leonti M, Raduner S, Racz I, Chen JZ, Xie XQ, Altmann KH, Karsak M and Zimmer A: Beta-caryophyllene is a dietary cannabinoid. Proc Nat Acad Sci USA 105: 9099-9104, 2008.

18. Ormeño E, Baldy V, Ballini C and Fernandez C: Production and diversity of volatile terpenes from plants on calcareous and siliceous soils: Effect of soil nutrients. J Chem Ecol 34: $1219-1229,2008$

19. Katsuyama S, Mizoguchi H, Kuwahata H, Komatsu T, Nagaoka K, Nakamura H, Bagetta G, Sakurada T and Sakurada S: Involvement of peripheral cannabinoid and opioid receptors in $\beta$-caryophyllene-induced antinociception. Eur J Pain 17: 664-675, 2013.

20. Paula-Freile LI, Andersen ML, Gama VS, Molska GR and Carlini EL: The oral administration of trans-caryophyllene attenuates acute and chronic pain in mice. Phytomedicine 21: 356-362, 2015 .

21. Chavan MJ, Wakte PS and Shinde DB: Analgestic and anti-inflammatory activity of caryophyllene oxide from Annona squamosa L. Bark. Phytomedicine 17: 149-151, 2010.

22. Martinez RM, Zarpelon AC, Cardoso RD, Vicentini FT, Georgetti SR, Baracat MM, Andrel CC, Moreira IC, Verri WA Jr and Casagrande R: Tephrosia sinapou ethyl acetate extract inhibits inflammatory pain in mice: Opioid receptor dependent inhibition of TNF $\alpha$ and IL-1 $\beta$ production. Pharm Biol 51: 1262-1271, 2013

23. Yamaguchi M, Weitzmann MN, Baile CA and Murata T: Exogenous regucalcin suppresses osteoblastogenesis and stimulates adipogenesis in mouse bone marrow culture. Integr Biol (Camb) 4: 1215-1222, 2012.

24. Yamaguchi M, Zhu S, Zhang S, Wu D, Moore TM, Snyder JP and Shoji M: Curcumin analogue UBS109 prevents bone loss in breast cancer bone metastasis mouse model: Involvement in osteoblastogenesis and osteoclastogenesis. Cell Tissue Res 357: 245-252, 2014.

25. Minkin C: Bone acid phosphatase: Tartrate-resistant acid phosphatase as a marker of osteoclast function. Calcif Tissue Int 34: 285-290, 1982.

26. Zaidi M, Blair HC, Moonga BS, Abe E and Huang CL: Osteoclastogenesis, bone resorption, and osteoblast-based therapeutics. J Bone Miner Res 18: 599-609, 2003. 